\title{
Adenocarcinoma Arising at a Urostomy: Case Report and Literature Review
}

\author{
Sungmi Jeon ${ }^{1,2}$ (D), Yujin Myung² (D), Changsik Pak Pa $^{2,3}$ \\ ${ }^{1}$ Department of Plastic and Reconstructive Surgery, Seoul National University Hospital, Seoul; ${ }^{2}$ Department of Plastic and Reconstructive Surgery, Seoul \\ National University Bundang Hospital, Seongnam; ${ }^{3}$ Department of Plastic Surgery, Asan Medical Center, Seoul, Korea
}

\begin{abstract}
We report a case of adenocarcinoma originating in a urostomy site 35 years after bladder cancer operation and urostomy formation. While ileostomy adenocarcinoma has been reported as a rare complication after colectomy and ileostomy formation for inflammatory bowel disease or familial adenomatous polyposis, there were no previously published cases of parastomal carcinoma in patients with urostomy. In our case, a series of work-ups, including immunohistochemical staining (cytokeratin 7 and 20, p63), revealed no evidence of primary adenocarcinoma of the skin or any other primary tumor. The patient underwent surgical excision with urostomy reformation and the skin defect was successfully reconstructed using local flap and split-thickness skin graft. This case poses a diagnostic challenge for clinicians because skin primary adenocarcinoma (i.e., malignant adnexal tumor) is likely to be ruled out due to its low incidence, and the symptoms may be considered those of a rare subcutaneous metastasis from a visceral malignancy (e.g., colon cancer) in the patient with a history of a prior malignancy (bladder cancer). This underscores the need for a multidisciplinary approach and patient education for early diagnosis.
\end{abstract}

Keywords: Adenocarcinoma; Skin neoplasms; Surgical wound; Urinary bladder

\section{Introduction}

Though primary urostomy adenocarcinoma has not been reported previously, here we report a case of an 80-year-old man who presented with nodular skin lesions and surrounding skin erosion on the right lower abdominal wall 35 years after a bladder cancer operation and urostomy formation. He was diagnosed with adenocarcinoma at the urostomy site. Because primary adenocarcinoma of the skin is extremely rare, additional work-up was required for accurate diagnosis and planning of treatment [1]. Immunohistochemical (IHC) marker staining figured substantially in conjunction with the patient's history, clinical presentation, and various imaging modalities, including computed tomography (CT) and ${ }^{18} \mathrm{~F}$-fluorodeoxyglucose (FDG) positron emission tomography (PET) [2]. Immunostaining of primary mucinous carcinoma of the skin, a rare malignant adnexal neoplasm of the eccrine gland, is positive for $\mathrm{cy}$ tokeratin 7 (CK7) and mucin, and negative for CK20 [3].

Although rare, cutaneous metastasis of internal malignancies should be also considered in the differential diagnosis, especially in patients with prior malignancy because it is often a factor for poor prognosis [4]. Metastatic skin cancer may originate from malignant melanoma, or from breast, lung, colon cancer and various other types of cancer and demonstrates nonspecific clinical findings, with pathology most commonly consistent with an adenocarcinoma [5].

In our case, there was no evidence of primary skin carcinoma or bladder urothelial

\section{Case Report}

Received: January 12, 2020

Revised: February 17, 2020

Accepted: February 29, 2020

Corresponding author: Changsik Pak, M.D., Ph.D.

Department of Plastic Surgery, Asan Medical Center, 88 Olympic-ro 43-gil, Songpa-gu,

Seoul 05505, Korea

Tel: +82-2-3010-3600

Fax: +82-2-476-7471

E-mail: iloveps@naver.com

Co-corresponding author: Yujin Myung, M.D., Ph.D.

Department of Plastic and Reconstructive Surgery, Seoul National University Bundang Hospital, 82 Gumi-ro 173beon-gil, Bundanggu, Seongnam 13620, Korea

Tel: +82-31-787-7229

Fax: +82-031-787-4055

E-mail: surgene@gmail.com

This is an Open Access article distributed under the terms of the Creative Commons Attribution Non-Commercial License (https://creativecommons.org/licenses/by-nc/4.0/) which permits unrestricted non-commercial use, distribution, and reproduction in any medium, provided the original work is properly cited.

C) 2020 Korean Wound Management Society 
carcinomas, and neither were other primary tumors found after a thorough medical history review, physical examination, detailed pathologic examination with IHC, various imaging studies (CT, PET), and colonoscopy. The adenocarcinoma originated at the skin-stoma junction, demonstrating locally invasive growth spreading into the adjacent epidermis. To the best of our knowledge, this is the first case report of adenocarcinoma arising at a urostomy site. The patient provided written informed consent for the publication and the use of his images.

\section{Case}

An 80-year-old man with a history of bladder cancer operation 35 years ago presented with two skin masses near the urostomy on the right lower abdomen (Fig. 1). He had changed an adhesive patch around the stoma (i.e., urostomy opening) every 5 days since the bladder cancer operation and urostomy formation. The ileal conduit had been made for urostomy, or urinary diversion, following cystectomy for bladder cancer. Approximately $15-20 \mathrm{~cm}$ of terminal ileum segment was used to serve as a reservoir for urine. The surrounding skin became erythematous 6 years ago. A protruding skin mass appeared on the erosive skin about 1 year ago and gradually increased in size. While changing the ostomy pouch, he noted a persistent peristomal skin lesion accompanied by protruding masses increasing in size and number, leading him to visit the dermatology clinic. There were two round nodular skin lesions with irregular surfaces sized approximately $2 \times 2 \mathrm{~cm}$, located close to- gether, at a $3-\mathrm{cm}$ distance inferior and lateral to the stoma. Approximately $10 \times 10 \mathrm{~cm}$ of the surrounding skin was diffusely erosive. The patient presented with no pain, tenderness, or other symptoms.

Dermatologists performed a punch biopsy, and all results were suggestive of an adenocarcinoma (Fig. 1B). A biopsyproven malignancy near the urostomy site was observed at the subcutaneous level on the right lower abdominal wall on CT scans. A $2.4 \times 1.9 \times 1.9-\mathrm{cm}$ enhancing nodule, not found in the prior biopsy (Fig. 2A), was also seen adjacent to the stoma. In the whole-body ${ }^{18} \mathrm{~F}$-FDG PET/CT imaging, an additional suspicious lesion with hypermetabolic uptake was detected within the tract of the urostomy site at a depth of $1.5 \mathrm{~cm}$ from the urostomy opening (Fig. 2B). It was later proven to be benign in the radiologists' needle biopsy. Otherwise, no abnormal lesion suggesting primary malignancy or any metastasis was found in other imaging studies, including chest CT. Serum tumor markers such as carcinoembryonic antigen (CEA) and cancer antigen (CA19-9) were measured, and test results were found to be within the normal range (CEA, $3.7 \mathrm{ng} / \mathrm{mL}$ [0-5]; CA19-9, $23.8 \mathrm{U} / \mathrm{mL}$ [0-27]).

According to IHC study, the adenocarcinoma showed CK20-positive and CK7-negative expression. In combination with negative p63 expression, the lesion was histologically diagnosed as adenocarcinoma rather than adenosquamous cell carcinoma, ruling out the possibility of urothelial carcinoma. CK7-negative and CK20-positive staining pattern correlates with the colorectal origin as the first etiology to be considered when diagnosing metastatic carcinomas of unknown primary
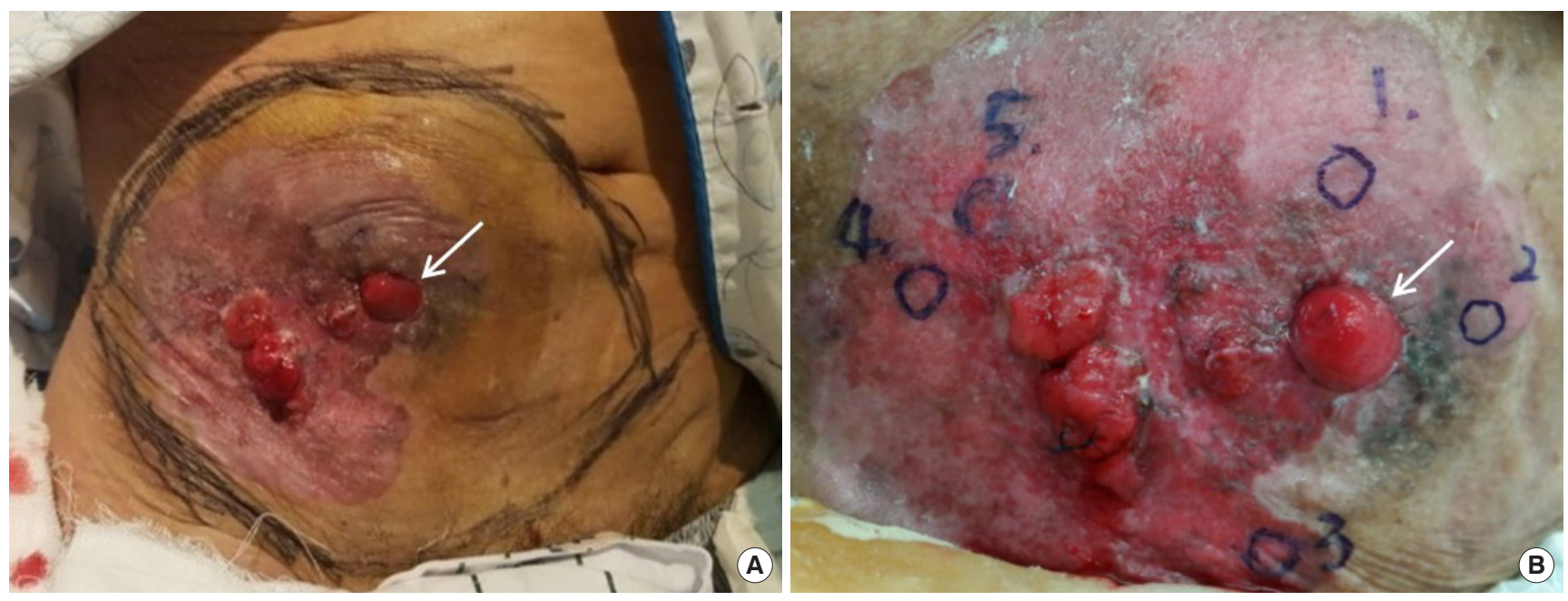

Fig. 1. Initial presentation of the patient. (A) Two $2 \times 2-\mathrm{cm}$ skin masses with protrusion and the surrounding erosive skin on the right abdominal wall. (B) Six encircled sites indicating where the biopsies were performed (white arrow: opening of the urostomy or stoma). 

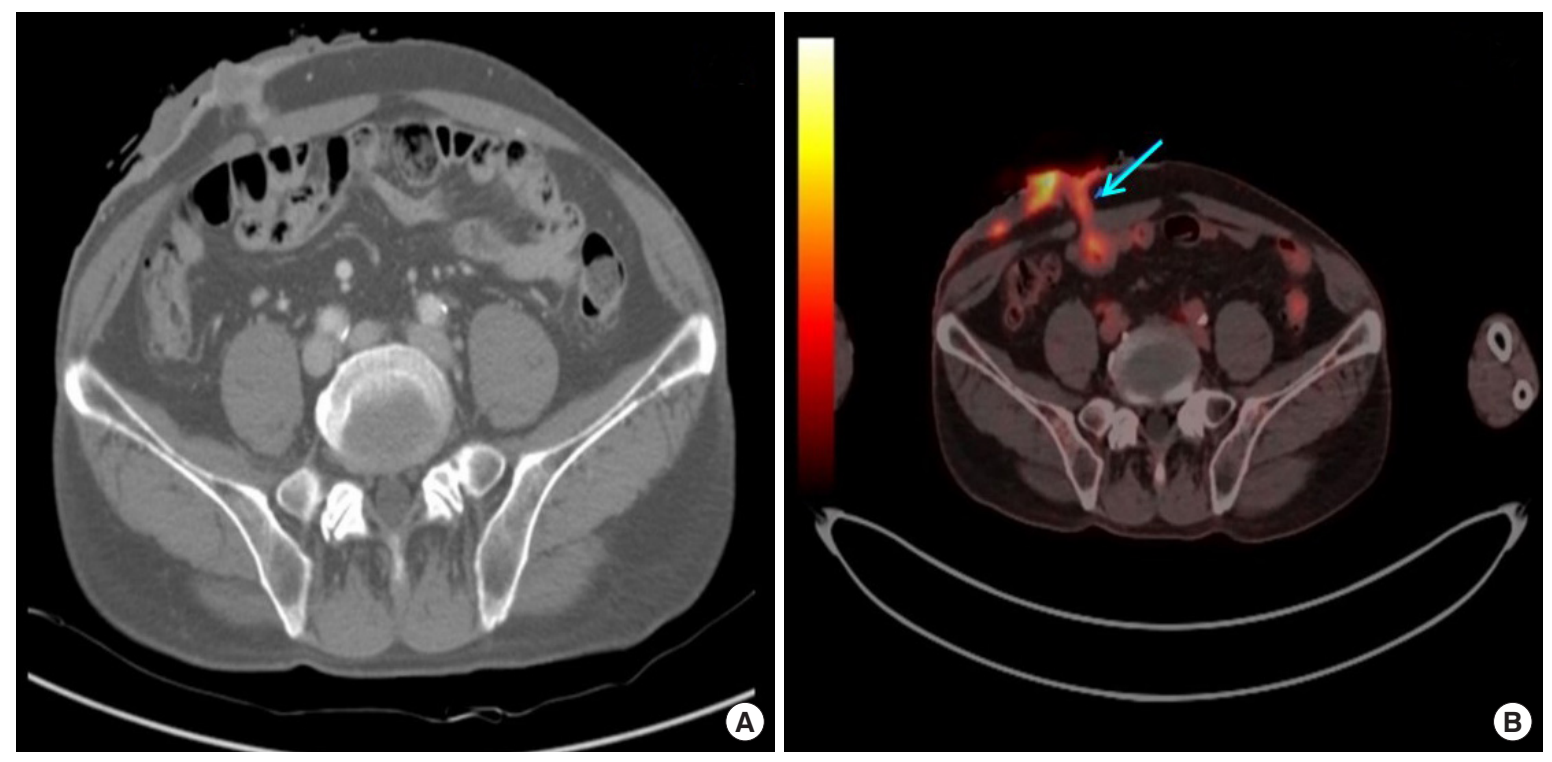

Fig. 2. Preoperative imaging evaluation to assess the lesion. (A) Axial contrast-enhanced computed tomography (CT) scan of the lower abdomen showing a $2.4 \times 1.9 \times 1.9-\mathrm{cm}$ enhancing nodule involving the stoma. (B) ${ }^{18} \mathrm{~F}$-fluorodeoxyglucose positron emission tomography/CT scan at the same anatomical level showing hypermetabolic uptake in the same parastomal mass and within the tract of the urostomy site (blue arrow).
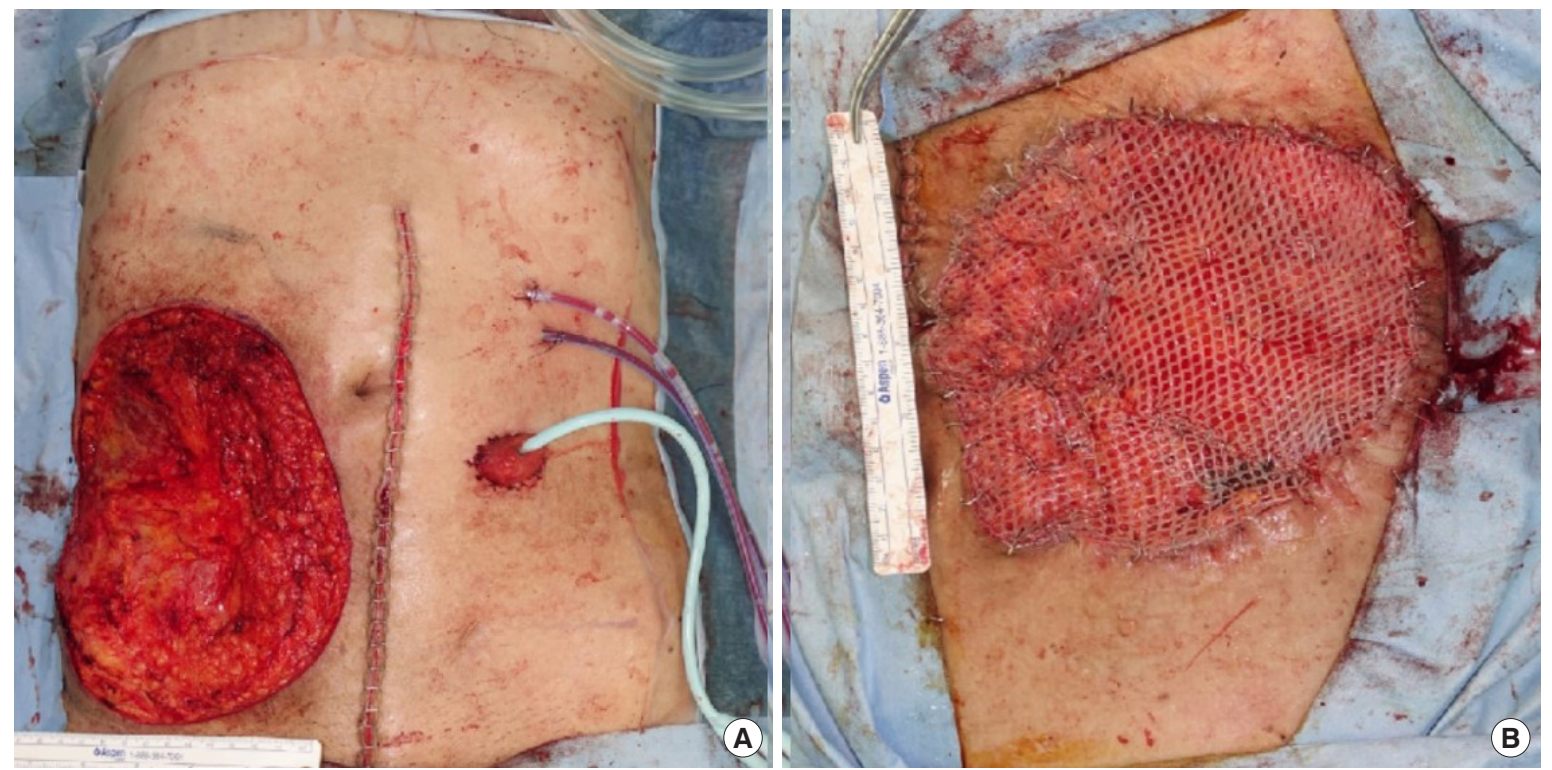

Fig. 3. Intraoperative and immediate postoperative images. (A) A $20 \times 15-\mathrm{cm}$ skin defect after wide excision of skin cancer and relocation of urostomy on the contralateral abdomen. (B) Split-thickness skin grafts applied after local flaps were raised to reduce the defect size.

sites [2]. A colonoscopy was performed to detect any suspected lesions in the lower gastrointestinal tract. There were two polyps in the distal descending and sigmoid colon $(40 \mathrm{~cm}$ and $20 \mathrm{~cm}$ from the anal verge, respectively), which were proven to be benign on biopsy.

Surgical excision was advised for the presumed metastatic skin lesion of unknown origin or primary adenocarcinoma. General surgeons performed a wide excision for the skin cancer with 1-2 cm margin and urostomy re-formation on the contralateral abdomen (Fig. 3A). They first tried a laparoscopic approach, but converted to open surgery using a midline incision because of severe abdominal adhesions found in the 

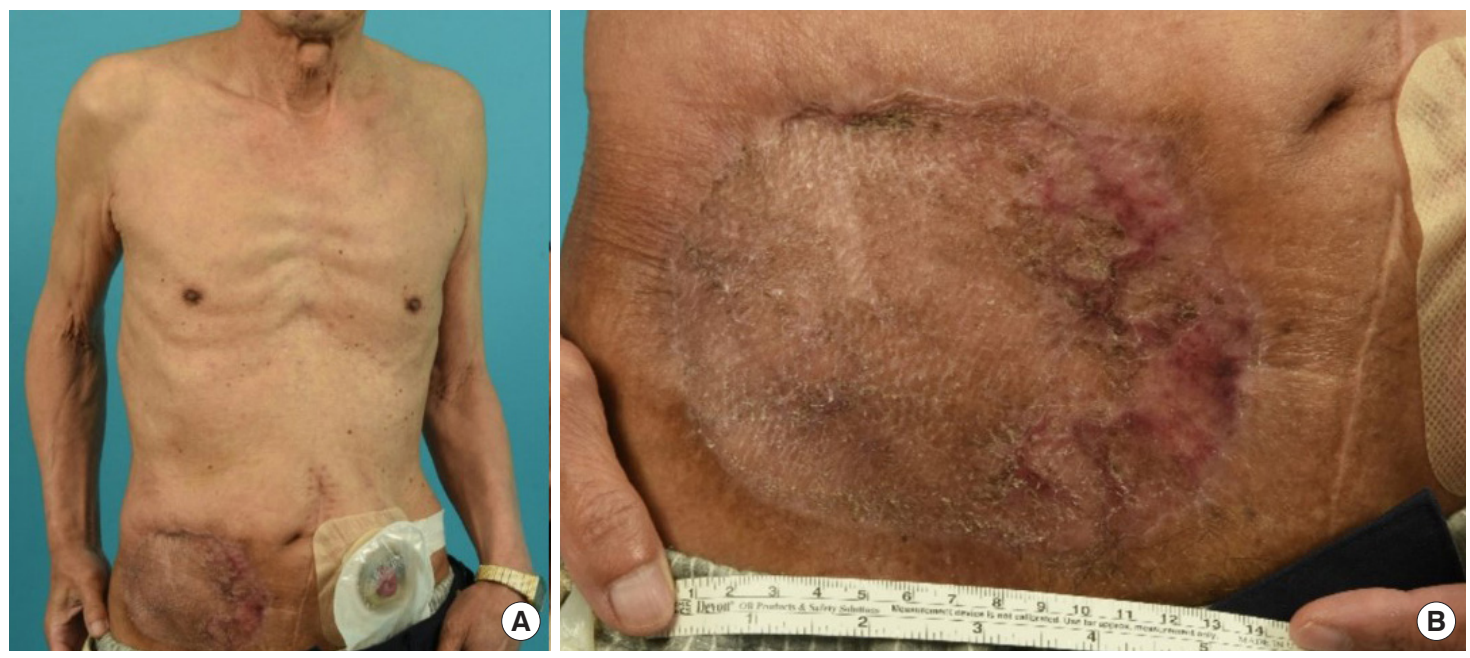

Fig. 4. Postoperative 3-month images. (A, B) The skin graft was well-taken without any complications.
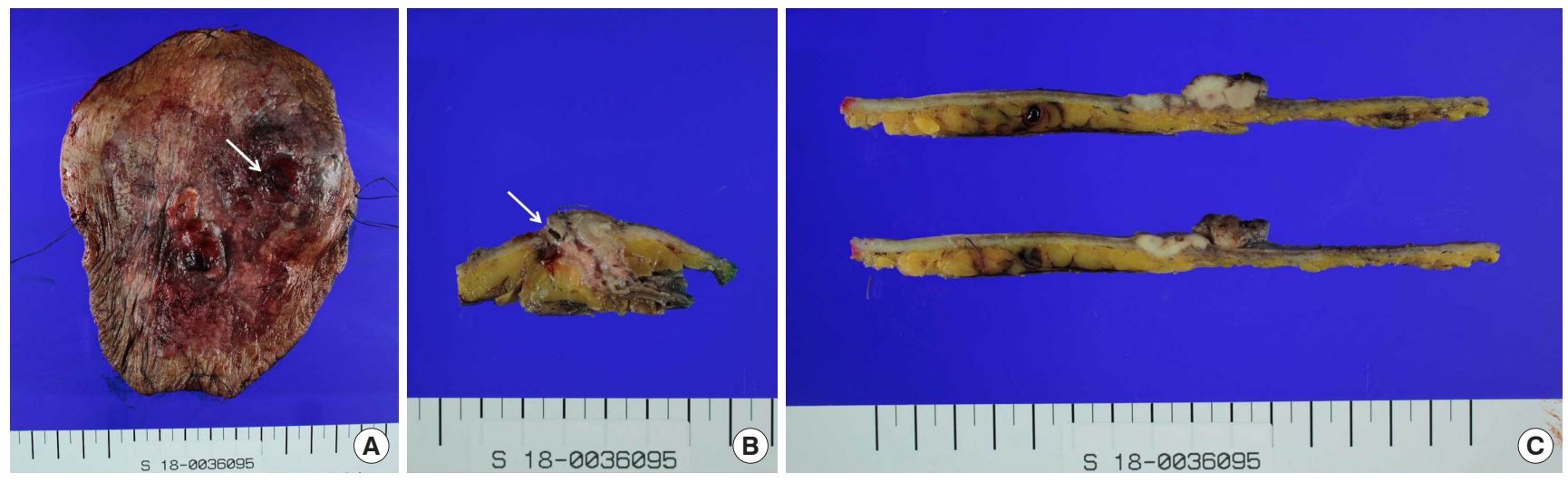

Fig. 5. Gross specimen photographs following wide excision. (A) Photograph of the entire fresh specimen prior to sectioning. (B) Photograph of histologic section showing a $2.4 \times 1.9 \times 1.9 \mathrm{~cm}$ nodule involving the skin-urostomy junction. (C) Photograph of section including the largest one $(2.2 \times 1.9 \times 1.2 \mathrm{~cm})$ among multiple nodules within the ulcerative lesion. White arrow is opening of the urostomy.

pelvis. After extensive adhesiolysis, two different segments of the injured small bowel underwent resection and anastomosis. The original urostomy was resected at the peritoneum level and its relocation to the left lower abdomen was limited because of adhesion. The new one was made by connecting the additional segment of terminal ileum to the remaining conduit in order to achieve sufficient conduit length. Afterwards maturation of the newly formed stoma was performed by urologists.

Reconstruction for the resultant skin defect on the right abdominal wall (size: $20 \times 15 \mathrm{~cm}$, wound bed: subcutaneous level) was requested of our plastic surgery department. After confirming the negative resection margin on frozen section, we performed local flap techniques in order to minimize the size of the defect and then applied a split-thickness skin graft (size: $12 \times 7 \mathrm{~cm}$, thickness: $12 / 1,000$ inch, 1:1.5 mesh, donor: bilateral thigh) (Fig. 3B). A vacuum-assisted closure dressing was applied to the skin graft for 5 days; the graft was successfully taken without complications (Fig. 4).

The pathology report of the surgical specimen diagnosed adenocarcinoma with pagetoid spread along epidermis, originating in the urostomy. On gross examination, the specimen contained multiple nodules beneath the diffusely dispersed skin ulceration, one of which was involved in the urostomy site (Fig. 5). The size of the entire lesion, including pagetoid spread, was measured $13.4 \times 11.4 \times 0.3 \mathrm{~cm}$. Pathological evaluation revealed that the peristomal skin lesions were pagetoid spread of infiltrative adenocarcinoma (Fig. 6). The spread was confined to the subcutaneous tissue (invasion depth: $4 \mathrm{~mm}$ ). There was no hemorrhage, necrosis, or calcification within the 

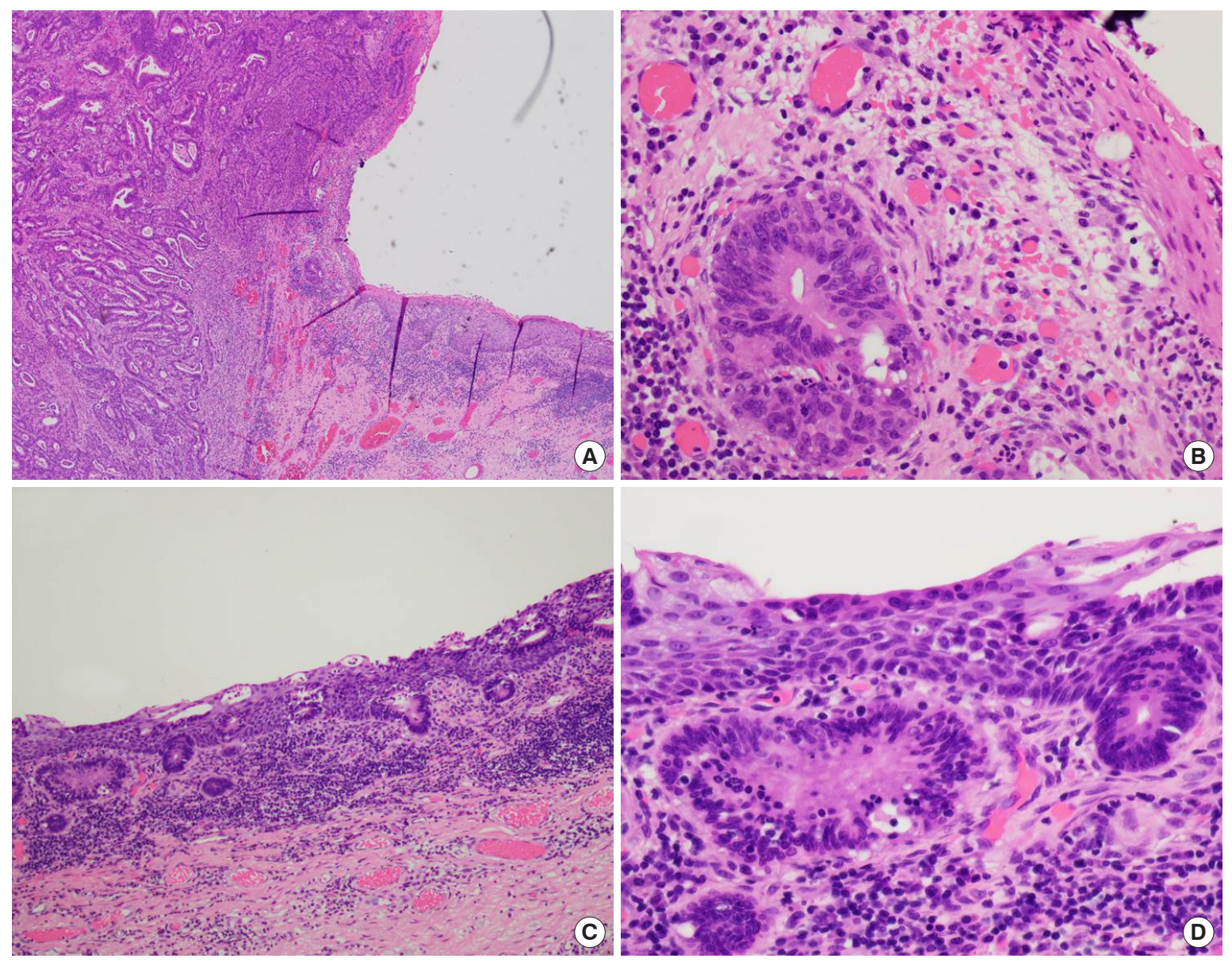

Fig. 6. Microscopic images of adenocarcinoma arising at the urostomy. Micrograph views showing that it was originated from the junction between the stoma and the skin (H\&E; A, $\times 40 ; B, \times 400$ ). Microscopic views showing pagetoid spread of infiltrative adenocarcinoma along the epidermis (H\&E; C, ×100; D, ×400).

nodules and neither were lympho-vascular and perineural invasion found. Surgical resection margins, $1.5 \mathrm{~cm}$ away from the mucosal margin of the excised stoma, were reported to be free of tumor cells.

After surgery, no adjuvant treatment was recommended. Regular follow-up was required to determine whether radiation therapy was necessary. Oncologists suggested that adjuvant chemotherapy could be considered in the case of local recurrence or distant metastasis in the future. The patient has received annual imaging follow-ups with concomitant clinical examinations, showing no recurrence or metastasis to date.

\section{Discussion}

We present the first case of adenocarcinoma arising at a urostomy site 35 years after urostomy formation. There had been no previously reported cases of parastomal carcinomas in pa- tients with urostomy. This case illustrated a diagnostic challenge for the clinicians because of the extremely low incidence of skin primary adenocarcinoma in general, the patient's history of a prior malignancy (bladder cancer), and the possibility of rare subcutaneous metastasis from a visceral malignancy (e.g., colon cancer). The original adenocarcinoma involved in the stoma might have readily progressed and locally spread into the same subcutaneous layer, leading to the dome-shaped skin nodules on the right lower abdominal wall that were first noticed by the patient.

Similarly, adenocarcinoma arising at an ileostomy site is a rare complication after colectomy and ileostomy formation for inflammatory bowel disease (ulcerative colitis, Crohn's disease) or familial adenomatous polyposis (FAP) [6-9]. The precise etiology of ileostomy carcinomas remains unclear, but colonic metaplasia from chronic inflammation has been considered to be correlated. Repetitive rounds of cellular prolifera- 
tion as a result of reparative processes at the mucocutaneous junction of an ileal conduit may play an important role in the pathogenesis of ileostomy carcinoma when considering the prolonged latent period following ileostomy formation (average of 30 years, as was our case). Furthermore, "backwash" ileitis in ulcerative colitis and a preexisting adenoma in FAP are attributed to the development of a precancerous condition called metaplasia. In our case, chronic exposure to irritating stool or urine, recurrent infections, and repeated trauma caused by using stomal adhesives might be causative.

According to the literature, ileostomy adenocarcinomas usually demonstrate fungating, exophytic growths involving the mucosa of the ileostomy [6-9]. Clinically, they can appear in various forms such as chronic ulcers or protruding masses. Variant presentations such as skin induration and irritation may pose a diagnostic challenge because most patients encounter simple peristomal skin complications that can be handled with adequate conservative management [10]. Newly developed lesions at stoma sites should be biopsied to rule out this rare malignancy. However, most skin issues can be managed by appropriate self-care measures (including gently handling the appliance securely fit around stomas, or utilizing adhesive remover/skin barrier powder). When managing the peristomal skin complications, regular physical exams are important for early detection.

Surgical excision and relocation of the stoma is the mainstay of treatment, as in ileostomy carcinomas [6-9]. Complete excision is regarded as a cure in almost all the cases. The resultant skin defects can be successfully reconstructed using splitthickness skin graft primarily or secondarily after the local flap is raised to decrease the size of the defect. A review of 36 primary adenocarcinomas at ileostomy sites by Metzger et al. [9] found that lymph node involvement was present in 19\% of cases with an $85 \%$ survival rate. Any suspicious skin lesions near stoma sites should be biopsied to rule out the possibility of this latent urostomy complication. Patient education and regular physician physical examinations are important for early detection.

Primary adenocarcinoma arising at a urostomy is very rare. The interval between the formation of a urostomy after bladder cancer operation and the diagnosis of adenocarcinoma at the urostomy site was 35 years in our case. This previously unreported case of urostomy adenocarcinoma is now presented to alert clinicians of its existence, emphasizing the need for a multidisciplinary approach and patient education for early diagnosis.

\section{Conflict of interest}

No potential conflicts of interest relevant to this article are reported.

\section{ORCID iDs}

Sungmi Jeon

https://orcid.org/0000-0001-9823-0469

Yujin Myung

https://orcid.org/0000-0001-5051-2440

Changsik Pak

https://orcid.org/0000-0002-3477-7120

\section{References}

1. Varadhachary GR, Abbruzzese JL, Lenzi R. Diagnostic strategies for unknown primary cancer. Cancer 2004;100: 1776-85.

2. Selves J, Long-Mira E, Mathieu MC, et al. Immunohistochemistry for diagnosis of metastatic carcinomas of unknown primary site. Cancers (Basel) 2018;10:108.

3. Maerki J, Ahmed S, Lee E. Primary mucinous carcinoma of the skin. Eplasty 2013;13:ic47.

4. Brownstein MH, Helwig EB. Patterns of cutaneous metastasis. Arch Dermatol 1972;105:862-8.

5. Llaguna $\mathrm{OH}$, Desai P, Fender AB, et al. Subcutaneous metastatic adenocarcinoma: an unusual presentation of colon cancer--case report and literature review. Case Rep Oncol 2010;3:386-90.

6. Procaccino L, Rehman S, Abdurakhmanov A, et al. Adenocarcinoma arising at ileostomy sites: two cases and a review of the literature. World J Gastrointest Surg 2015;7:94-7.

7. Quah HM, Samad A, Maw A. Ileostomy carcinomas a review: the latent risk after colectomy for ulcerative colitis and familial adenomatous polyposis. Colorectal Dis 2005;7:53844.

8. Ejtehadi F, Nizamoglu M, Sivakumar R. Squamous cell carcinoma at an ileostomy site-fifty-four years following colectomy for ulcerative colitis: a case report and literature review. Int J Surg Case Rep 2013;4:678-80.

9. Metzger PP, Slappy AL, Chua HK, et al. Adenocarcinoma developing at an ileostomy: report of a case and review of the literature. Dis Colon Rectum 2008;51:604-9.

10. Doctor K, Colibaseanu DT. Peristomal skin complications: causes, effects, and treatments. Chronic Wound Care Manag Res 2017;4:1-6. 